Reference for this article:

Salmond, C. H., Meiser-Stedman, R., Glucksman, E., Thompson, P., Dalgleish, T., \& Smith, P. (2011). The nature of trauma memories in acute stress disorder in children and adolescents. Journal of Child Psychology and Psychiatry, 52(5), 560-570.

This is the author post-print.

If you would like the published pdf, just email one of the authors or co-authors (e.g. r.meiserstedman@uea.ac.uk, patrick.smith@kcl.ac.uk, tim.dalgleish@mrc-cbu.cam.ac.uk) 


\title{
The nature of trauma memories in acute stress disorder in children and adolescents
}

\author{
C.H. Salmond, ${ }^{1}$ R. Meiser-Stedman, ${ }^{1}$ E. Glucksman, ${ }^{2}$ P. Thompson, ${ }^{2}$ T. Dalgleish,${ }^{3}$ \\ \& P. Smith ${ }^{1}$
}

\author{
${ }^{1}$ Department of Psychology, Institute of Psychiatry, King's College London, UK \\ ${ }^{2}$ Emergency Department, King's College Hospital, London, UK; \\ ${ }^{3}$ Medical Research Council Cognition and Brain Sciences Unit, Cambridge, UK
}

Background: There is increasing theoretical, clinical and research evidence for the role of trauma memory in the aetiology of acute pathological stress responses in adults. However, research into the phenomenology of trauma memories in young people is currently scarce.

Methods: This study compared the nature of trauma narratives to narratives of unpleasant non-traumatic events in young people (aged 8-17) who sought emergency medical attention following an assault or road traffic accident. Data were collected within 2-4 weeks of the index event. Symptom severity was assessed by child self-report and face-to-face diagnostic interviews. Comparisons of narrative indices were made between those children with acute stress disorder (ASD) and those without ASD.

Results: Among participants $(\mathrm{n}=50)$, those with ASD $(38 \%)$ had significantly elevated levels of disorganisation in their trauma narrative, compared both to trauma-exposed controls and to their unpleasant comparative narrative. This effect was not accounted for by age. Regardless of ASD diagnostic status, trauma narratives had significantly higher sensory content and significantly lower positive emotion content compared to the unpleasant comparative narrative. These effects were not significant when age was included as a covariate. Acute symptom severity was significantly predicted by the level of disorganisation in the trauma narrative and the child's cognitive appraisals of the event.

Conclusions: These data provide the first empirical evidence that disorganisation is not only directly linked to symptom severity, but also specific to the trauma memory. In addition, it provides support for the adaptation of adult cognitive models to acute pathological stress reactions in children and adolescents. Keywords: Trauma, adolescence, memory, pathology.

Abbreviations: ASD: Acute stress disorder; PTSD: post traumatic stress disorder; RTA: road traffic accident; ED: emergency department.

Brief title: Trauma memory in acute stress disorder

Conflict of interest statement: No conflicts declared. 


\section{Introduction}

Recent cognitive models of pathological stress responses have emphasised the role of trauma memory characteristics in the aetiology of posttraumatic stress disorder (PTSD). Two cognitive theories argue that peritraumatic influences, such as data-driven processing or heightened arousal, disrupt normal encoding and lead to fragmented memories (Ehlers \& Clark, 2000; Foa \& Rothbaum, 1998). A third cognitive theory argues that trauma memories are over-represented in a separate sensory-based memory system and comparatively underrepresented in verbally accessible memories (Brewin, Dalgleish, \& Joseph, 1996; Dalgleish, 2004).

Empirical studies of trauma memory functioning in adults have employed self-report questionnaire methods, alongside the potentially more robust and objective method of analysing trauma narratives. Three key characteristics of trauma memories have been identified. Among adults with PTSD and acute stress disorder (ASD), trauma memories are fragmented and disorganised; have prominent sensory features; and show increased emotional content (e.g., Ehlers \& Steil, 1995; Engelhard, van den Hout, Kindt, Arntz, \& Schouten, 2003; Foa, Molnar, \& Cashman, 1995; Halligan, Michael, Clark, \& Ehlers, 2003; Koss, Figueredo, Bell, Tharan, \& Tromp, 1996; Rubin, Feldman, \& Beckham, 2004).

The literature on trauma memory quality in young people is limited and has relied heavily on self-report questionnaire data (see Dalgleish, Meiser-Stedman, \& Smith, 2005). Studies using single item questions (Ehlers, Mayou, \& Bryant, 2003; Stallard \& Smith, 2007) have produced inconsistent findings. When a more comprehensive self-report questionnaire was used, young people with pathological stress responses rated their trauma memory as having greater sensory features compared to traumaexposed controls (McKinnon, Nixon, \& Brewer., 2008; Meiser-Stedman, Smith, Glucksman, Yule, \& Dalgleish, 2007). Use of self-report measures to characterise memory quality has been criticised because responses may be influenced by demand characteristics (Pasupathi, 2007) and meta-memory (Kindt \& van den Hout, 2003). Some argue that it is paradoxical to obtain self-ratings of memory quality from individuals deemed to have memory deficits (O’Kearney \& Perrott, 2006).

Few studies with young people have used the alternative method of collecting and analysing trauma narratives to measure memory characteristics. Such studies have tended to focus on the accuracy and completeness of narratives of traumatic or stressful events (e.g., Fivush \& Sales, 2006; Peterson, Sales, Rees, \& Fivush, 2007). To date, only one study has investigated the relationship between trauma narratives and traumatic stress responses in young people. Kenardy et al. (2007) reported that disorganisation of narratives (but not degree of emotional content) was related to traumatic stress reactions among 7-15-year-olds who had been admitted to hospital. This study has a number of limitations. The analysis used dichotomous categories rather than continuous measures. Symptom severity was determined by parental report, which corresponds poorly to child report (e.g., Ellis, Stores, \& Mayou, 1998; Meiser-Stedman et al., 2008). Developmental changes in narrative skill or episodic memory such as improved organisation and reduced repetition were not controlled for (e.g., Gathercole, 1998). Finally, the study did not include a comparison narrative in order to determine the specificity of any trauma narrative characteristics. This is pertinent because developmental research shows that children provide more organised reports of negative events than neutral or emotionally positive events (Ackil, Van Abbema, \& Bauer, 2003; Fivush, 1998; Fivush, Hazzard, Sales, Sarfati, \& Brown, 2003).

The current study therefore sought to extend these important initial findings by Kenardy et al., by examining predictors of a diagnosis of acute stress disorder (ASD) in the aftermath of trauma in a sample of children and adolescents recruited from an emergency department. In the present study, 
trauma narratives were collected and analysed in detail to derive a number of continuous indices of memory quality. Non-trauma narratives were also collected in order to test for the specificity of any memory effects. Developmental differences in episodic memory (e.g., Gathercole, 1998) were controlled for by including age as a covariate in all analyses. Symptom severity was assessed by child self-report and face-to-face diagnostic interviews.

A number of previous studies have identified demographic, cognitive, and family factors which increase the risk of developing symptoms of PTSD and ASD. For example, female gender, prior exposure to trauma, and elevated subjective threat appraisal at the time of exposure increase the risk of ASD in adults, although the findings in children are less consistent (e.g., Harvey \& Bryant, 1999; MeiserStedman, Dalgleish, Smith, Yule, \& Glucksman, 2007). The same factors, plus a previous history of mental health difficulties and cognitive misappraisals of the trauma and its consequences, have been shown to increase the risk for PTSD in both adults and children, with an additional factor of parental mental health problems increasing risk for PTSD in children (Meiser-Stedman, Dalgleish, Glucksman, Yule, \& Smith, 2009; Pine \& Cohen, 2002). The current study therefore measured these previously established factors in order to attempt to replicate previous findings, and to examine whether memory characteristics also predict acute stress symptomatology over and above these previously established factors.

The following hypotheses were investigated:

1. In line with previous studies, young people with ASD will have more pre-trauma emotional and behavioural difficulties; elevated subjective threat appraisal; increased cognitive misappraisals of the trauma and its consequences; and more symptomatic parents compared to the non-ASD controls.

2. The memory of the trauma of young people with ASD will be characterised by increased disorganisation, emotional content and sensory information, both when compared to the trauma memory of trauma-exposed non-ASD controls and when compared to their own non-trauma memory for negative events. These differences will remain when age and degree of distress at the time of recollection are subsequently included in the analyses.

3. Regression modelling will reveal that those trauma memory characteristics identified as important under Hypothesis 2 will predict overall degree of acute stress symptomatology, independent of the influence of any significant demographic and trauma-related variables identified under Hypothesis 1.

\section{Methods}

Young people aged 8-17 years who were consecutive attendees at a London Emergency Department (ED) following an assault or road traffic accident (RTA) were invited to take part. Exclusion criteria: severe learning disability, organic brain injury, involvement of social services and inability to speak English (assessed from the young people's ED records and when first in contact with families). Potential participants were sent a letter within a week of attendance at ED, followed up by a telephone call within a few days. If families agreed to participate, a home visit was arranged within 2-4 weeks of the child's trauma. Participants were rewarded with shopping vouchers. This study was given ethical approval (ref 06/Q0706/110) and informed consent was obtained from carers and children.

\section{Measures}


Objective indices of trauma severity and demographic information were collected from ED records and via interview with families. UK standard categorisation of socioeconomic status was used, based on parental occupation (NS-SEC; Office for National Statistics, 2005).

Parents completed the Strengths and Difficulties Questionnaire (SDQ; Goodman, 1997) based on child behaviour in the six months prior to their trauma. Parents were specifically asked to provide an estimate of their child's prior behavioural difficulties rather than any symptoms which had evolved since the trauma. Parents also completed the Hospital Anxiety and Depression Scale (Zigmond \& Snaith, 1983) as a measure of current parental anxiety and depression.

Young people completed four self-report questionnaires of peritraumatic processing and posttraumatic symptoms. Subjective severity of threat at the time of the trauma was assessed with a three-item measure ('I really thought that I was going to die', 'I was really scared' and 'I thought that I was going to be very badly hurt'). Extent of agreement was rated on a 4-point scale, with higher scores representing stronger agreement. Internal reliability is satisfactory (alpha $=.69$, study sample).

Severity of acute stress symptoms was assessed using the Child Post-Traumatic Stress Scale (CPSS; Foa, Johnson, Feeny, \& Treadwell, 2001), a 17-item self-report questionnaire which directly measures PTSD symptoms as defined in DSM-IV. Symptom frequency in the last two weeks is rated as 'Not at all', 'Once a week or less', '2 to 4 times a week' and ' 5 or more times a week'. These responses score 0 , 1,2 and 3, respectively, and a total score is calculated. The measure possesses good internal consistency (alpha $=.89$; study sample). It should be noted that the CPSS does not comprehensively cover the dissociation symptoms that are part of the ASD criteria.

Maladaptive appraisals were assessed with the 25-item Children's Posttraumatic Cognitions Inventory (CPTCI; Meiser-Stedman, Smith, et al., 2009). Extent of agreement with items (e.g., 'My life has been destroyed by the frightening event', 'My reactions since the frightening event show that I must be going crazy') is rated on a 4-point scale, with higher scores indicating greater maladaptive appraisals. Internal reliability is good (alpha $=.90$, study sample).

Symptoms of depression were assessed using the Birleson Depression Self-Rating Scale (DSRS; Birleson, 1997), an 18-item self-report measure, rated on a 3-point scale. The measure has good internal reliability (alpha $=.70$, study sample).

Young people were interviewed face-to-face using the PTSD module of the child version of the Anxiety Disorders Interview Schedule for DSM-IV (ADIS; Silverman \& Albano, 1996) (with supplementary dissociative questions to facilitate diagnosis of ASD; Meiser-Stedman, Dalgleish, et al., 2007).

\section{Trauma memory}

Participants were asked to give a detailed verbal narrative of their traumatic event, using child-adapted instructions (Kenardy et al. 2007). Participants were not prompted for further details once they had begun their narrative. Following Halligan et al.'s (2003) method, participants ranked any distress in response to the recall task on a $0-100$-point visual analogue scale. Participants provided a second narrative of a recent unpleasant event (which occurred prior to the traumatic event), using similar procedures. If the child struggled to identify an appropriate event, suggestions of possible events were given (e.g., argument with a friend, watching a scary film, a school exam; based on work in control populations). The order in which these two narratives were recalled was counter-balanced between participants. Narratives were recorded digitally, transcribed verbatim, and coded according to the 
phrase-based method described by Foa et al. (1995). The narrative was segmented into phrases and each phrase was categorised. Frequencies of utterances in each category were converted to percentages to control for differences in narrative length. Coding was carried out by raters who were blind to all other measures, including symptom severity scores. A disorganisation index (Halligan et al., 2003) was calculated from three measures from the Foa coding: clauses consisting of repetitions; clear expressions of uncertainty, confusion, or non-consecutive chunks; clauses indicating understanding of what happened (reversed). Each score was z-transformed, and a summary memory disorganisation score was calculated (Halligan et al., 2003). The sensory, positive feelings, negative feelings and angry feelings indices from the Foa analysis were also used. Each narrative was assigned a global coherence rating from 0 (not at all disorganised) to 10 (extremely disorganised). A random sample of 10 transcripts was blindly rated by a second rater and a further sample of 10 transcripts was rated twice by the same rater. Interand intra-rater reliability for the Foa analysis were .94 and .99 , respectively, and for the global coherence were .87 and .81 respectively.

Participants also completed the Trauma Memory Quality Questionnaire (TMQQ; Meiser-Stedman, Smith, Yule, \& Dalgleish, 2007), separately, in relation to both the trauma memory and the unpleasant memory. The TMQQ is an 11-item self-report measure of the visual quality (e.g., 'My memories of the frightening event are mostly pictures or images'), degree of verbal encoding (e.g., 'I can't seem to put the frightening event into words'), temporal context (e.g., 'When I remember the frightening event I feel like it is happening right now'), and nonvisual sensory quality (e.g., 'When I think about the frightening event I can sometimes smell things that I smelt when the frightening event happened') of the memories for an event. Items are rated on a 4-point scale, with higher scores indicating memories that are more sensory and less verbally accessible. Internal consistency is good (alpha > .68; study sample). In summary, self-report measures and coding of narratives resulted in seven independent variables of memory quality for each narrative: the Halligan index of disorganisation; sensory, positive feelings, negative feelings and angry feelings indices; a global coherence measure; and the TMQQ.

\section{Participants}

One hundred and seventy-eight young people met the criteria for entry. Of these, 24 (13\%) could not be reached on the telephone number recorded in their notes, $98(55 \%)$ declined to take part and $56(31 \%)$ agreed to take part. Of the 56 families who agreed, 50 (28\% of the total meeting the inclusion criteria) were able to attend an initial assessment session.

Participants $(n=50)$ did not differ from non-participants $(n=128)$ in terms of sex or the proportion of children arriving in an ambulance. Participants were significantly younger. Those with a 'very urgent' (yellow) triage rating (i.e., a nurse's rating of how urgently treatment was needed) and assault victims were found to be under-represented in the participants group compared to non-participants (see Table 1). All participants who had been assaulted were physically attacked by other adolescents (some known and some unknown to the victim).

\section{Demographic, trauma and symptom variables (see Table 2)}

Nineteen children (38\% of the sample) met criteria for acute stress disorder on the supplemented ADISC. The ASD group and Non ASD $(n=31)$ group did not differ in terms of age, gender, ethnicity, socioeconomic level, whether their parent was present at the traumatic event, prior contact with mental health services, triage level, ambulance arrival, admission, days since event, type of event (assault or RTA) or details of event (e.g., pedestrian or weapon used) ( $p>$.05). The mean NS-SEC (an estimate of socioeconomic level) score for the sample was 2.24, ranging from 1 to 5, indicating that most participants' parents were in 'intermediate occupations' (i.e., not managerial occupations or routine 
occupations). Over half the participants classified their ethnicity as Black and around $40 \%$ as Caucasian. Half the participants were male and the participants' average age was 13.5 years. Unsurprisingly, the ASD group reported significantly more symptoms of traumatic stress and depression compared to the Non ASD group.

\section{Statistical analysis}

An alpha level of .05, two-tailed, was used throughout. The Kolmogorov-Smirnov statistic was used to determine whether the data met the assumption of normality for parametric statistics and box plots were used to detect variables with outliers. When the Kolmogorov-Smirnov statistic was significant or outliers were found, the data were then re-analysed using non-parametric statistics. The pattern of the results did not vary using non-parametric statistics, so for clarity, only parametric statistics are reported.

\section{Results}

\section{Hypothesis 1: ASD vs non-ASD comparisons}

We found broad support for our first hypothesis in that young people with ASD showed more pretrauma emotional difficulties on the SDQ; elevated subjective threat appraisal; increased cognitive misappraisals of the trauma and its consequences on the CPTCI compared to the non-ASD controls (see Table 2). However, the mean score of the ASD group for emotional problems on the SDQ was nevertheless below the clinical cut-off of 5 and there were no significant group differences on the remaining SDQ subscales. We found no support for our prediction that parents of the ASD group would show elevated anxiety or depression on the HADS.

\section{Hypothesis 2: Memory comparisons}

As outlined in the introduction, we analysed our various indices of trauma memory quality initially without age and distress covaried, and then assessed whether any effects remained significant when these covariates were included. Initial analyses also included narrative order (unpleasant or trauma first) as a factor but there were no significant main effects or interactions involving order and so analyses without order included are reported here. In light of the preliminary nature of this study, an analytically conservative approach was taken when investigating this hypothesis. A MANOVA for the whole sample was carried out, with diagnostic status of child (ASD and Non ASD) and event (unpleasant and trauma) as independent variables, and with the seven narrative indices (the Halligan index of disorganisation; sensory, positive feelings, negative feelings and angry feelings indices; a global coherence measure; and the TMQQ) as dependent measures. The multivariate output indicated a significant main effect of event type (Wilk's Lambda $=.52, \mathrm{~F}(7,42)=5.48, \mathrm{p}<.001$, partial eta square $=.48$ ) and a trend towards an effect of diagnostic status (Wilk's Lambda $=.748, \mathrm{~F}(7,42)=2.01, \mathrm{p}=.076$, partial eta square $=.25$ ). Critically, these were qualified by a significant interaction between event and diagnostic status (Wilk's Lambda $=.70, \mathrm{~F}(7,42)=2.60, \mathrm{p}=.025$, partial eta square $=.30$ ). This critical interaction between event and diagnostic status remained significant after covarying age, Wilk's Lambda $=.70, F(7,41)=2.53$, $p$ $=.03$, partial eta squared $=.30$, and near-significant when covarying distress, Wilk's Lambda $=.75, \mathrm{~F}(7$, $41)=1.98, p=.08$, partial eta squared $=.25$. There remained trend effects for diagnostic status and event after inclusion of the covariates, $P$ s between .07 and .13 .

In light of these multivariate effects, we examined the univariate ANOVA output for the seven narrative indices (see Table 3 for a summary), again initially without age and distress as covariates and then again with these factors covaried. 
Trauma Memory Quality Questionnaire (TMQQ). On the child-completed TMQQ, comparisons between the trauma and unpleasant event scores revealed significant main effects of event and diagnostic group, and a trend towards a significant interaction between event and diagnostic group. The ASD group scored higher (indicating that the memories are rated as more sensory and fragmented) than the Non ASD group on both the TMQQ completed for the trauma and the TMQQ completed for the unpleasant event.

Trauma narratives. Analysis of the Halligan index of disorganisation revealed a significant interaction between event narrative and diagnostic group, with no significant main effects of either diagnostic group or event narrative. Follow-up analysis revealed that this interaction reflected a significant difference between the event narratives in the ASD group (with a higher disorganisation index for the trauma narrative compared to the unpleasant narrative) and, interestingly, a trend to the reverse effect in the Non-ASD group (see Figure 1; paired t-test: ASD: $t=2.22, \mathrm{p}=.04$, Non ASD: $\mathrm{t}=1.88, \mathrm{p}=.07$ ). ANCOVA analysis revealed that neither age nor levels of distress associated with recall of the event rendered the interaction non-significant (Fs > 7.31).

Analysis of the global coherence rating also revealed a significant interaction between event narrative and diagnostic group and a significant main effect of event narrative, but no significant main effect of diagnostic group (see Table 3). Follow-up analysis revealed that the interaction reflected a significant difference between the event narratives in the ASD group (with a higher score [i.e., lower coherence] for the trauma narrative compared to the unpleasant narrative) and a non-significant difference in the Non ASD group (paired t-test: ASD: $\mathrm{t}=3.88, \mathrm{p}<.01$, Non ASD: $\mathrm{t}=1.63, \mathrm{p}=.12$ ). ANCOVA revealed that age did not account for this interaction, $\mathrm{F}=4.49, \mathrm{p}=.04$. However, when level of distress experienced during narration was included as a covariate the interaction was reduced to a trend, $\mathrm{F}=2.69, \mathrm{p}=.11$. Analysis of the sensory index revealed a main effect of event narrative, but no significant main effect of diagnostic group or interaction between event narrative and diagnostic group. The trauma narratives contained significantly more sensory phrases than the unpleasant narratives. When age was included as a covariate, this was no longer significant, $\mathrm{F}<1$. To explore this further, the participants were divided into two groups around median age $(14.35)$, the Younger $(n=25)$ and Older $(n=25)$ groups. The younger participants showed wider variation in the amount of sensory information included in their trauma narrative (Younger group: Trauma narrative 3.09 (6.97) (mean, SD); Unpleasant narrative .42 (1.29), Older group: Trauma narrative 2.30 (2.20); Unpleasant narrative .42 (1.70)) but the numbers are too small in the separate age cells to merit a focused statistical analysis.

Results from the emotional indices revealed a significant main effect of event narrative for the positive feelings index, but no significant main effect of diagnostic group nor an interaction. The trauma narratives contained significantly fewer positive feelings segments compared to the unpleasant narratives. When age was included as a covariate, there was no longer a significant effect of event narrative, $F(1,47)=2.19, p=.15$. The younger participants showed wider variation in the amount of positive emotion information included in their trauma narrative (Younger group: Trauma narrative $0(0)$ (mean, SD); Unpleasant narrative 1.14 (3.49), Older group: Trauma narrative .17 (.64); Unpleasant narrative .77 (1.91)). No significant main effects or interactions were found for the negative feelings or angry feelings indices.

\section{Hypothesis 3: Regression modelling}

To examine the independent effects of our measures of trauma memory quality, after covarying other significant trauma-related and demographic factors we used regression modelling. To do this we used our continuous measure of acute stress symptomatology, the CPSS, as there was insufficient study power for a robust logistic regression model involving ASD diagnostic status. In an initial stage of 
variable reduction we examined zero-order correlations between the following putative predictors of CPSS levels: age; gender; previous mental health services contact; previous trauma; subjective threat of trauma; CPTCI total; TMQQ; Birleson depression scale; Halligan index of disorganisation; positive feelings index; negative feelings index; angry feelings index; sensory index; parental mental health, as indexed by the parental HADS Depression and parental HADS Anxiety sub-scales; and SDQ total problems [parentrated] (see Table 4).

We then performed a linear regression with CPSS as the dependent variable and entering all significantly related zero-order predictors of the CPSS in a forward stepwise manner (see Table 5). The final model accounted for $70.9 \%$ of variance in scores on the CPSS, with CPTCI total and the Halligan index of disorganisation accounting for unique variance in CPSS scores.

\section{Discussion}

The results from the current study revealed that young people with a diagnosis of ASD showed greater pre-existing emotional problems, higher subjective threat appraisal at time of their trauma exposure, and reported more cognitive misappraisals than trauma-exposed non-ASD controls. The trauma narratives produced by the participants with ASD showed greater disorganisation relative to narratives of other unpleasant events; this pattern was not seen in those without ASD. Finally, regression modelling revealed that the level of this trauma narrative disorganisation (along with the degree of maladaptiveness of cognitive appraisals about the trauma and its consequences) predicted the severity of the acute stress response, independent of other relevant trauma-related and demographic factors.

These results provide the first empirical evidence to our knowledge that disorganised memory is not only related to the presence of a stress disorder in young people but is also specific to the memory of the trauma. This extends the findings in the adult literature (Foa et al., 1995; Halligan et al., 2003; Harvey \& Bryant, 1999; Jones, Harvey, \& Brewin, 2007) and initial findings in young people (Kenardy et al., 2007). The data are consistent with cognitive models of trauma responses (Ehlers \& Clark, 2000; Foa \& Rothbaum, 1998) and emphasise the importance of targeting such memories in the treatment of stress disorders in youth (Smith et al., 2007). The disorganised trauma narratives do not appear to be simply a reflection of dissociative amnesia: 27 young people reported not remembering important aspects of their trauma, and only 14 were in the ASD group. The results from this study do not provide clear support for the predictions of cognitive models of stress disorders (Brewin et al., 1996; Ehlers \& Clark, 2000) that would predict differential saturation with sensory information in the trauma narratives in those with ASD. This is consistent with null empirical findings from the adult literature (Jones et al., 2007; Zoellner, Alvarez-Conrad, \& Foa, 2002). Further interpretation of this finding would be premature due to the small sample size and effect of including age as a covariate on the findings. Developmentally informed research, with a larger sample of children across a range of ages, is needed in order to determine the importance of sensory aspects of memory in children.

The absence of a significant relationship between emotional indices and ASD status in the current study is also consistent with results from both the adult (Alvarez-Conrad, Zoellner, \& Foa, 2001; Harvey \& Bryant, 1999; Jones et al., 2007; Zoellner et al., 2002; though see Manne et al., 2002) and child literatures (Kenardy et al., 2007). This finding may reflect attempts by those experiencing clinically significant post-trauma stress responses to control their affect when narrating the trauma. Narratives may vary according to the purpose of the narration (Ehlers \& Clark, 2000) and material that is particularly personal, emotional or shaming may not be included in the absence of a containing therapeutic 
relationship. Alternatively, it may simply be that the affective quality of a trauma memory is not sufficient per se to produce the distinctive re-experiencing symptoms of ASD.

Trauma memories were rated on the TMQQ as significantly more sensory and fragmented in nature than unpleasant memories, for all individuals regardless of diagnostic status, in line with findings from the narrative analysis. Furthermore, participants with ASD rated memories overall as more sensory and fragmented than participants without ASD. This suggests that subjects' perceptions of the nature of their memories recollected in the dayto-day remembering as assessed on the TMQQ are potentially different to the impression derived from objective analysis of their narratives. Whether this reflects a genuine difference between day-to-day remembering and narrative-driven recollection is difficult to say due to the study's limited power and the different means by which the data were collected, with the retrospective questionnaire and narrative approaches potentially being differentially influenced by symptom severity, subsequent processing, demand characteristics or meta-memory (Brewin \& Holmes, 2003; Kindt \& van den Hout, 2003; O’Kearney \& Perrott, 2006).

The severity of the acute pathological stress reactions - early PTSD symptoms - was significantly predicted by the Halligan index of disorganisation and the CPTCI. This finding is consistent with the Ehlers and Clark (2000) model and builds on previous research in young people which has highlighted the importance of these two factors individually in symptom severity (Kenardy et al., 2007; MeiserStedman, Dalgleish, et al., 2007; Salmon, Sinclair, \& Bryant, 2007). The tentative clinical implications of these findings are that psychological treatments for youth with significant acute stress reactions should include components addressing the cognitive processing of the trauma. In particular, such interventions should target the structure and organisation of the trauma memory and the meaning of the trauma.

\section{Limitations and strengths of the study}

A number of study limitations should be noted. The recruitment rate was low. Participants also differed from non-participants in terms of age and triage category, and fewer assault victims participated in the study. As such it is possible that the study sample has a lower severity of disorder than would be found in the wider trauma-exposed population. For instance, it is highly plausible that those individuals with high avoidance symptoms would actively choose not to participate in a study that explicitly asks them to discuss their trauma. Additionally, ED attendees were contacted within a week of their trauma, and it is therefore possible that their decision to participate was influenced by the nature of their (or their family members') response to the trauma. However, the participation rate and pattern of recruitment are similar to previous studies (e.g., McDermott \& Cvitanovich, 2000; Stallard, 2003; Stallard \& Smith, 2007). Nevertheless, future studies are needed to establish the generalisability of these findings, as it is possible that the lack of effects observed is attributable to biases in the sample (such as lower severity of disorder). Generally, the small sample size reduced the power for complex statistical models and may have limited the study's ability to fully examine the effect of age on patterns observed.

While the use of narrative analysis was a strength of the study, the child's given narrative of events will not be a complete and accurate measure of the actual memory representation. Narratives may be influenced by difficulties with retrieval and expression (Brewin \& Holmes, 2003), a reluctance to discuss the trauma (McNally, 2005) or current levels of trauma symptoms (Bryant \& Harvey, 1999; Southwick, Morgan, Nicolaou, \& Charney, 1997). Indeed, it is not possible to determine the direction of causality between the narratives and symptom severity from this study. 
Despite this, there are a number of strengths to the current study, including: obtaining reports from children directly in face-to-face interviews and via standardised questionnaires; deriving multiple indices of memory characteristics via questionnaire and detailed narrative coding; use of a comparison narrative to determine the specificity of findings to trauma memories; and analysis using both a continuous measure of early PTSD symptoms as well as ASD diagnostic criteria.

\section{Future research directions}

A number of areas for future investigation are indicated. The current sample was limited to children over the age of 7. In light of the cognitive and linguistic developments that occur prior to the age of 7 (e.g., Gathercole, 1998; Yasik, Saigh, Oberfield, \& Halamandaris, 2007), future studies should investigate the applicability of the findings from this study to younger children. This is particularly pertinent in light of the age effects seen in the sensory and emotional indices. The developmental literature has identified that parental coping styles, attachment patterns and reminiscing styles influence children's narratives (Fivush \& Sales, 2006; Peterson et al., 2007; Sales \& Fivush, 2005). Future studies should investigate the relationships between traumatic stress symptoms and factors such as family processes (including reminiscing style and coping strategies) and narratives. The sample of young people in this study experienced relatively mild injury and their traumatic events did not have the widespread consequences associated with largerscale events (such as hurricanes). Although the diagnostic criteria for ASD are not trauma specific (American Psychiatric Association, 2000), future studies should confirm that the results are generalisable to other trauma populations. Additionally, studies with larger samples should investigate any potential moderating effect of trauma type and symptoms presentation (in particular the influence of avoidance on trauma narrative).

Finally, this study focused on the relationship between acute symptoms and narrative quality. Future studies should investigate the influence of these early indices on chronic symptom severity. This may provide important indicators of increased risk of significant chronic difficulties and identify potential therapeutic targets for treatment.

\section{Conclusion}

Disorganisation of the trauma narrative, but not its sensory or emotional content, was significantly higher than for narratives of unpleasant, nontrauma events in young people with ASD. This pattern was not seen in those without ASD. Acute symptom severity was predicted by the level of narrative disorganisation and by maladaptive cognitive appraisals about the trauma and its sequelae. These data provide the first empirical evidence that narrative disorganisation is specific to the trauma memory in youth and support the adaptation of adult cognitive models to acute pathological stress reactions in children and adolescents (Meiser-Stedman, 2002). 


\section{Acknowledgements}

The authors would like to thank Dr Daniel Stahl (Institute of Psychiatry, KCL) for statistical advice and John Poynton, Gulgun Dandeniya and Brenda Moss (Emergency Department, King's College Hospital) for support with recruitment.

\section{Correspondence to}

Claire Salmond, Department of Psychology, Addiction Sciences Building, Institute of Psychiatry, 4 Windsor Walk, London SE5 8AF, UK; Tel: +44 207848 0224; Fax: +44 0207848 0860; Email: claire.salmond@iop.kcl.ac.uk

\section{Key points}

- Adult cognitive models highlight a role of the nature of trauma memory representations in the aetiology of acute pathological stress responses in adults.

- This study shows that young people with acute stress disorder have more disorganised trauma narratives compared to trauma-exposed controls, and compared to their narratives of other unpleasant events.

- Overall symptom severity was independently significantly predicted by the level of disorganisation in the trauma narrative, and by the maladaptiveness of the child's cognitive appraisals of the event.

- These data provide support for the adaptation of adult cognitive models to acute pathological stress reactions in children and adolescents. 


\section{References}

Ackil, J.K., Van Abbema, D.L., \& Bauer, P.J. (2003). After the storm: Enduring differences in motherchild recollections of traumatic and nontraumatic events. Journal of Experimental Child Psychology, 84, 286-309.

Alvarez-Conrad, J., Zoellner, L.A., \& Foa, E.B. (2001). Linguistic predictors of trauma pathology and physical health. Applied Cognitive Psychology, 15, 159-170.

American Psychiatric Association. (2000). Diagnostic and statistical manual of mental disorders (DSMIV-TR) (4th edn, text rev.). Washington, DC: Author.

Birleson, P. (1997). The Birleson Depression Scale. In I. Sclare (Ed.), Child psychology portfolio (pp. 43-44). Windsor: NFER-Nelson.

Brewin, C.R., Dalgleish, T., \& Joseph, S. (1996). A dual representation theory of posttraumatic stress disorder. Psychological Review, 103, 670-686.

Brewin, C.R., \& Holmes, E.A. (2003). Psychological theories of posttraumatic stress disorder. Clinical Psychology Review, 23, 339-376.

Bryant, R.A., \& Harvey, A.G. (1999). Acute stress disorder: A handbook of theory, assessment and treatment. Washington, DC: American Psychological Association.

Dalgleish, T. (2004). Cognitive theories of posttraumatic stress disorder: The evolution of multirepresentational theorizing. Psychological Bulletin, 130, 228-260.

Dalgleish, T., Meiser-Stedman, R., \& Smith, P. (2005). Cognitive aspects of posttraumatic stress reactions and their treatment in children and adolescents: An empirical review and some recommendations. Behavioural and Cognitive Psychotherapy, 33, 459-486.

Ehlers, A., \& Clark, D.M. (2000). A cognitive model of posttraumatic stress disorder. Behaviour Research and Therapy, 38, 319-345.

Ehlers, A., Mayou, R.A., \& Bryant, B. (2003). Cognitive predictors of posttraumatic stress disorder in children: Results of a prospective longitudinal study. Behaviour Research and Therapy, 41, 1-10.

Ehlers, A., \& Steil, R. (1995). Maintenance of intrusive memories in posttraumatic stress disorder: A cognitive approach. Behavioural and Cognitive Psychotherapy, 23, 217-249.

Ellis, A., Stores, G., \& Mayou, R. (1998). Psychological consequences of road traffic accidents in children. European Child and Adolescent Psychiatry, 7, 61-68.

Engelhard, I.M., van den Hout, M.A., Kindt, M., Arntz, A., \& Schouten, E. (2003). Peritraumatic dissociation and posttraumatic stress after pregnancy loss: A prospective study. Behaviour Research and Therapy, 41, 67-78.

Fivush, R. (1998). Children's recollections of traumatic and nontraumatic events. Development and Psychopathology, 10, 699-716.

Fivush, R., Hazzard, A., Sales, J.M., Sarfati, D., \& Brown, T. (2003). Creating coherence out of chaos? Children's narratives of emotionally positive and negative events. Applied Cognitive Psychology, 17, 1-19.

Fivush, R., \& Sales, J.M. (2006). Coping, attachment, and mother-child narratives of stressful events. Merrill-Palmer Quarterly, 52, 125-150.

Foa, E.B., Johnson, K.M., Feeny, N.C., \& Treadwell, K.R.H. (2001). The Child PTSD Symptom Scale: A preliminary examination of its psychometric properties. Journal of Clinical Child Psychology, 30, 376-384.

Foa, E.B., Molnar, C., \& Cashman, L. (1995). Change in rape narratives during exposure therapy for posttraumatic stress disorder. Journal of Traumatic Stress, 8, 675-690.

Foa, E.B., \& Rothbaum, B.O. (1998). Treating the trauma of rape: Cognitive behavioural therapy for PTSD. New York: Guilford Press. 
Gathercole, S.E. (1998). The development of memory. Journal of Child Psychology and Psychiatry, 39, $3-27$.

Goodman, R. (1997). The Strengths and Difficulties Questionnaire: A research note. Journal of Child Psychology and Psychiatry, 38, 581-586.

Halligan, S.L., Michael, T., Clark, D.M., \& Ehlers, A. (2003). Posttraumatic stress disorder following assault: The role of cognitive processing, trauma memory, and appraisals. Journal of Consulting and Clinical Psychology, 71, 419-431.

Harvey, A.G., \& Bryant, R.A. (1999). A qualitative investigation of the organization of traumatic memories. British Journal of Clinical Psychology, 38, 401-405.

Jones, C., Harvey, A.G., \& Brewin, C.R. (2007). The organisation and content of trauma memories in survivors of road traffic accidents. Behaviour Research and Therapy, 45, 151-162.

Kenardy, J., Smith, A., Spence, S.H., Lilley, P.-R., Newcombe, P., Dob, R., et al. (2007). Dissociation in children's trauma narratives: An exploratory investigation. Journal of Anxiety Disorders, 21, 456466.

Kindt, M., \& van den Hout, M. (2003). Dissociation and memory fragmentation: Experimental effects on meta-memory but not on actual memory performance. Behaviour Research and Therapy, 41, 167178.

Koss, M.P., Figueredo, A.J., Bell, I., Tharan, M., \& Tromp, S. (1996). Traumatic memory characteristics: A cross-validated mediational model of response to rape among employed women. Journal of Abnormal Psychology, 105, 421-432.

Manne, S., DuHamel, K., Nereo, N., Ostroff, J., Parsons, S., Martini, R., et al. (2002). Predictors of PTSD in mothers of children undergoing bone marrow transplantation: The role of cognitive and social processes. Journal of Pediatric Psychology, 27, 607-617.

McDermott, B.M., \& Cvitanovich, A. (2000). Posttraumatic stress disorder and emotional problems in children following motor vehicle accidents: An extended case series. Australian and New Zealand Journal of Psychiatry, 34, 446-452.

McKinnon, A.C., Nixon, R., \& Brewer, N. (2008). The influence of data-driven processing on perceptions of memory quality and intrusive symptoms in children following traumatic events. Behaviour Research and Therapy, 46, 766-775.

McNally, R.J. (2005). Debunking myths about trauma and memory. Canadian Journal of Psychiatry Revue Canadienne de Psychiatrie, 50, 817-822.

Meiser-Stedman, R. (2002). Towards a cognitive-behavioral model of PTSD in children and adolescents. Clinical Child and Family Psychology Review, 5, 217-232.

Meiser-Stedman, R., Dalgleish, T., Glucksman, E., Yule, W., \& Smith, P. (2009). Maladaptive cognitive appraisals mediate the evolution of posttraumatic stress reactions: A 6-month follow up of child and adolescent assault and motor vehicle accident survivors. Journal of Abnormal Psychology, 188, 778787.

Meiser-Stedman, R., Dalgleish, T., Smith, P., Yule, W., \& Glucksman, E. (2007). Diagnostic, demographic, memory quality and cognitive variables associated with acute stress disorder in children and adolescents. Journal of Abnormal Psychology, 116, 65-79.

Meiser-Stedman, R., Smith, P., Bryant, R.A., Salmon, K., Yule, W., Dalgleish, T., et al. (2009). Development and validation of the Child Post-Traumatic Cognitions Inventory (CPTCI). Journal of Child Psychology and Psychiatry, 50, 432- 440.

Meiser-Stedman, R., Smith, P., Glucksman, E., Yule, W., \& Dalgleish, T. (2007). Parent and child agreement for acute stress disorder, post-traumatic stress disorder and other psychopathology in a prospective study of children and adolescents exposed to single-event trauma. Journal of Abnormal Child Psychology, 35, 191-201. 
Meiser-Stedman, R., Smith, P., Glucksman, E., Yule, W., \& Dalgleish, T. (2008). The post-traumatic stress disorder (PTSD) diagnosis in pre-school and elementary school-aged children exposed to motor vehicle accidents. American Journal of Psychiatry, 165, 1326-1337.

Meiser-Stedman, R., Smith, P., Yule, W., \& Dalgleish, T. (2007). The Trauma Memory Quality Questionnaire: Preliminary development and validation of a measure of trauma memory characteristics for children and adolescents. Memory, 15, 271-279.

O'Kearney, R., \& Perrott, K. (2006). Trauma narratives in posttraumatic stress disorder: A review. Journal of Traumatic Stress, 19, 81-93.

Office for National Statistics. (2005). The National Statistics Socio-economic Classification user manual. London: HMSO.

Pasupathi, M. (2007). Telling and the remembered self: Linguistic differences in memories for previously disclosed and previously undisclosed events. Memory, 15, 258-270.

Peterson, C., Sales, J.M., Rees, M., \& Fivush, R. (2007). Parent-child talk and children's memory for stressful events. Applied Cognitive Psychology, 21, 1057-1075.

Pine, D.S., \& Cohen, J.A. (2002). Trauma in children and adolescents: Risk and treatment of psychiatric sequelae. Biological Psychiatry, 51, 519-531.

Rubin, D.C., Feldman, M.E., \& Beckham, J.C. (2004). Reliving, emotions and fragmentation in the autobiographical memories of veterans diagnosed with PTSD. Applied Cognitive Psychology, 18, 1735 .

Sales, J.M., \& Fivush, R. (2005). Social and emotional functions of mother-child reminiscing about stressful events. Social Cognition, 23, 70-90.

Salmon, K., Sinclair, E., \& Bryant, R.A. (2007). The role of maladaptive appraisals in child acute stress reactions. British Journal of Clinical Psychology, 46, 203-210.

Silverman, W.K., \& Albano, A.M. (1996). Anxiety Disorders Interview Schedule for DSM-IC: Child and Parent Interview Schedule. San Antonio, TX: Psychological Corporation.

Smith, P., Yule, W., Perrin, S., Tranah, T., Dalgleish, T., \& Clark, D. (2007). Cognitive behavior therapy for PTSD in children and adolescents: A preliminary randomized controlled trial. Journal of the American Academy of Child and Adolescent Psychiatry, 46, 1051-1061.

Southwick, S.M., Morgan, C.A., 3rd, Nicolaou, A.L., \& Charney, D.S. (1997). Consistency of memory for combat-related traumatic events in veterans of Operation Desert Storm [see comment]. American Journal of Psychiatry, 154, 173-177.

Stallard, P. (2003). A retrospective analysis to explore the applicability of the Ehlers and Clark (2000) cognitive model to explain PTSD in children. Behavioural and Cognitive Psychotherapy, 31, $337-$ 345.

Stallard, P., \& Smith, E. (2007). Appraisals and cognitive coping styles associated with chronic posttraumatic symptoms in child road traffic accident survivors. Journal of Child Psychology and Psychiatry, 48, 194-201.

Yasik, A.E., Saigh, P.A., Oberfield, R.A., \& Halamandaris, P.V. (2007). Posttraumatic stress disorder: Memory and learning performance in children and adolescents. Biological Psychiatry, 61, 382-388.

Zigmond, A.S., \& Snaith, R.P. (1983). The Hospital Anxiety and Depression Scale. Acta Psychiatrica Scandinavica, 67, 361- 370.

Zoellner, L.A., Alvarez-Conrad, J., \& Foa, E.B. (2002). Peritraumatic dissociative experiences, trauma narratives, and trauma pathology. Journal of Traumatic Stress, 15, 49-57. 
Table 1. Characteristics of participants and non-participants.

\begin{tabular}{|c|c|c|c|c|c|}
\hline \multirow[b]{2}{*}{ Categorical variables } & \multicolumn{2}{|c|}{ Frequency $(\%)$} & \multicolumn{3}{|c|}{ Statistics } \\
\hline & $\begin{array}{c}\text { Participants } \\
n=50\end{array}$ & $\begin{array}{c}\text { Non-participants } \\
\mathrm{n}=128\end{array}$ & $\chi^{2}$ & df & $\mathrm{p}$ \\
\hline Gender (male) & $25(50.00)$ & $79(62.17)$ & 2.21 & 1 & .14 \\
\hline Trauma type & & & 3.79 & 1 & .05 \\
\hline Assault & $30(60.00)$ & $95(74.22)$ & & & \\
\hline RTA & $20(40.00)$ & $32(25.00)$ & & & \\
\hline Missing data & 0 & $1(.88)$ & & & \\
\hline Triage & & & ${ }^{\#} 13.88$ & 6 & .03 \\
\hline 4-Green & $39(78.00)$ & $82(64.06)$ & & & \\
\hline 3 - Orange & $0(.00)$ & $2(1.56)$ & & & \\
\hline $2-$ Yellow & $4(8.00)$ & $26(20.31)$ & & & \\
\hline $1-\operatorname{Red}$ & $3(6.00)$ & $5(3.91)$ & & & \\
\hline Resus & $3(6.00)$ & $5(3.91)$ & & & \\
\hline Missing data & $1(2.00)$ & $7(5.47)$ & & & \\
\hline Ambulance arrival & $18(36.00)$ & $55(42.97)$ & 1.15 & 1 & .28 \\
\hline Missing data & $2(4.00)$ & $9(7.03)$ & & & \\
\hline & \multicolumn{2}{|c|}{ Mean $(\mathrm{SD})$} & \multicolumn{3}{|c|}{ Statistics } \\
\hline Continuous variables & $\begin{array}{c}\text { Participants } \\
\mathrm{n}=50\end{array}$ & $\begin{array}{c}\text { Non-participants } \\
\mathrm{n}=128\end{array}$ & $\mathrm{~F}$ & df & $\mathrm{p}$ \\
\hline Age & $13.50(2.51)$ & $14.72(2.18)$ & 10.32 & 1,176 & .002 \\
\hline
\end{tabular}

Note: Triage category represents a nurse's rating of how urgently the individual needs treatment: a triage category of 1 (or red) indicates immediate treatment required, 2 (or yellow) indicates very urgent treatment, 3 (or orange) indicates urgent treatment, and 4 (or green) indicates standard treatment. 'Resus' indicates that the participant bypassed the triage system and received immediate emergency treatment. ${ }^{\#}$ Likelihood ratio. 
Table 2. Group comparison of mean (SD) (or frequency (\%) for categorical data) of symptom and demographic measures

\begin{tabular}{|c|c|c|c|c|c|}
\hline Measure & ASD $n=19$ & Non ASD $\mathrm{n}=31$ & $\mathrm{~F}$ & df & $\mathrm{p}$ \\
\hline \multicolumn{6}{|l|}{ Demographic measures } \\
\hline Age & $13.32(2.69)$ & $13.61(2.43)$ & $<1$ & & \\
\hline Gender (male) & $7(36.84)$ & $18(58.06)$ & 2.12 & 1 & .15 \\
\hline Ethnicity & & & ${ }^{\#} 4.64$ & 4 & .33 \\
\hline Black African & $3(15.79)$ & $2(6.45)$ & & & \\
\hline Black Caribbean & $2(10.53)$ & $4(12.90)$ & & & \\
\hline Black Other & $4(21.11)$ & $10(32.26)$ & & & \\
\hline Other & $0(.00)$ & $3(9.68)$ & & & \\
\hline Caucasian & $9(47.37)$ & $12(38.71)$ & & & \\
\hline Missing data & $1(5.26)$ & $0(.00)$ & & & \\
\hline \multicolumn{6}{|l|}{ Trauma characteristics } \\
\hline Parent present at trauma & $3(15.79)$ & $5(16.13)$ & $\#_{<.01}$ & 1 & .98 \\
\hline Triage level & & & \#3.70 & 3 & .30 \\
\hline 4-Green & $17(89.47)$ & $22(7.97)$ & & & \\
\hline 3 - Orange & $0(0)$ & $0(0)$ & & & \\
\hline $2-$ Yellow & $1(5.26)$ & $3(9.68)$ & & & \\
\hline $1-$ Red & $1(5.26)$ & $2(6.45)$ & & & \\
\hline Resus & $0(0)$ & $3(9.68)$ & & & \\
\hline Missing data & $0(0)$ & $1(3.23)$ & & & \\
\hline Ambulance arrival & $7(36.84)$ & $11(35.48)$ & .02 & 1 & .88 \\
\hline Admitted & $4(21.11)$ & $4(12.90)$ & ${ }^{\#} .57$ & 1 & .45 \\
\hline Days since event & $20.74(4.27)$ & $21.48(3.67)$ & $<1$ & & \\
\hline Type of event (RTA) & & & 2.39 & 1 & .12 \\
\hline RTA & $5(26.32)$ & $15(48.39)$ & & & \\
\hline Assault & $14(73.68)$ & $16(51.61)$ & & & \\
\hline \multicolumn{6}{|l|}{ Detail of event } \\
\hline \multicolumn{6}{|l|}{ Assault } \\
\hline Weapon used & $2(14.29)^{Z}$ & $6(37.50)^{Z}$ & $\# 4.60$ & 2 & .10 \\
\hline Assailant known to participant & $8(57.14)^{Z}$ & $8(50.00)^{\mathrm{Z}}$ & .15 & 1 & .67 \\
\hline RTA & & & \# 2.37 & 4 & .67 \\
\hline Bicycle hit by motor vehicle & $0(0)^{\mathrm{Y}}$ & $1(6.67)^{Y}$ & & & \\
\hline Fall from bicycle & $0(0)^{Y}$ & $1(6.67)^{Y}$ & & & \\
\hline Passenger in motor vehicle & $3(60.00)^{Y}$ & $5(33.33)^{Y}$ & & & \\
\hline Motorbike rider & $0(.00)^{\mathrm{Y}}$ & $1(6.67)^{Y}$ & & & \\
\hline Pedestrian & $2(40.00)^{Y}$ & $7(46.67)^{Y}$ & & & \\
\hline \multicolumn{6}{|l|}{ Symptom measures (reported by child) } \\
\hline CPSS total & $24.95(7.17)$ & $11.23(8.13)$ & 36.61 & 1,48 & $<.01$ \\
\hline Birleson Depression Scale & $15.16(8.65)$ & $6.16(3.91)$ & 18.27 & $1,22.6$ & $<.01$ \\
\hline CPTCI total & $57.00(15.00)$ & $40.32(9.69)$ & 18.70 & $1,27.3$ & $<.01$ \\
\hline Subjective threat severity & $9.63(2.24)$ & $7.97(2.46)$ & 5.77 & 1,48 & .02 \\
\hline
\end{tabular}

Parental symptoms 
HADS Anxiety

HADS Depression

Parental rating of child emotional and behavioural difficulties prior to trauma

SDQ Total

SDQ emotional problems

SDQ Conduct problems

SDQ Hyperactivity

SDQ Peer problems

Prior contact with mental health
$10.26(4.47)$

$5.84(3.32)$

$13.11(5.89)$

$4.11(2.75)$

$2.32(1.63)$

$4.32(2.38)$

$2.37(2.31)$

2 (10.53)
$7.90(5.08)$

$4.79(4.71)$

2.73

1,46

$<1$

$9.37(6.87)$

3.84

12.32

1,47

$1.73(1.98)$

$1.80(2.11)$

$3.90(2.55)$

1.93 (1.82)

2 (6.45)

$<1$

$<1$

$<1$

\#.23

.64

Note: ${ }^{\#}$ Likelihood ratio. CPSS: Child Post-Traumatic Stress Scale; CPTCI: Children's Posttraumatic Cognitions Inventory; SDQ: Strengths and Difficulties Questionnaire; HADS: Hospital Anxiety and Depression Scale. For details of triage codes, see Table 1.

${ }^{\mathrm{Z}}$ Percentages are percent of participants in ASD and non-ASD groups who experienced assaults $(\mathrm{n}=14$ and 16 respectively).

${ }^{\mathrm{Y}}$ Percentages are percent of participants in ASD and non-ASD groups who experienced an RTA ( $\mathrm{n}=5$ and 15 respectively). 
Table 3. Mean (SD) and ANOVA output of TMQQ and narrative indices.

\begin{tabular}{|c|c|c|c|c|c|}
\hline Index & ASD & Non ASD & Event & Diagnosis & Interaction \\
\hline \multicolumn{6}{|l|}{ TMQQ } \\
\hline Trauma narrative & $28.26(6.09)$ & $23.29(4.69)$ & $\mathrm{F}(1,48)=5.79, \mathrm{p}=.02$ & $F(1,48)=6.30, p=.02$ & $\mathrm{~F}(1,48)=2.81, \mathrm{p}=.10$ \\
\hline Unpleasant narrative & $24.84(6.40)$ & $22.68(5.82)$ & & & \\
\hline \multicolumn{6}{|c|}{ Halligan index of disorganisation } \\
\hline Trauma narrative & $.64(2.17)$ & $-.39(1.60)$ & $\mathrm{F}<1$ & $\mathrm{~F}<1$ & $F(1,48)=9.76 p<.01$ \\
\hline Unpleasant narrative & $-.57(1.86)$ & $.35(1.73)$ & & & \\
\hline \multicolumn{6}{|l|}{ Coherence rating } \\
\hline Trauma narrative & $5.47(2.89)$ & $3.84(2.60)$ & & & \\
\hline Unpleasant narrative & $3.16(2.19)$ & $3.10(2.37)$ & $\mathrm{F}(1,48)=16.73, \mathrm{p}<.01$ & $\mathrm{~F}(1,48)=1.81, \mathrm{p}=.18$ & $F(1,48)=4.43, p=.04$ \\
\hline \multicolumn{6}{|l|}{ Sensory } \\
\hline Trauma narrative & $\begin{array}{l}1.66 \\
(2.19)\end{array}$ & $3.33(6.25)$ & & & \\
\hline Unpleasant narrative & $.44(1.91)$ & $.40(1.21)$ & $\mathrm{F}(1,46)=7.17 \mathrm{p}=.01$ & $\mathrm{~F}<1$ & $\mathrm{~F}<1$ \\
\hline \multicolumn{6}{|l|}{ Positive feelings } \\
\hline Trauma narrative & $.15(.67)$ & $.05(.26)$ & & & \\
\hline Unpleasant narrative & $1.87(4.23)$ & $.39(1.08)$ & $F(1,48)=6.55, p=.01$ & $F(1,48)=3.88, p=.06$ & $F(1,48)=2.89, p=.1$ \\
\hline \multicolumn{6}{|l|}{ Negative feelings } \\
\hline Trauma narrative & $5.52(10.64)$ & $2.39(5.07)$ & & & \\
\hline Unpleasant narrative & $4.57(4.02)$ & $3.79(5.81)$ & $\mathrm{F}<1$ & $\mathrm{~F}(1,48)=1.80, \mathrm{p}=.20$ & $\mathrm{~F}<1$ \\
\hline \multicolumn{6}{|l|}{ Angry feelings } \\
\hline Trauma narrative & $.00(.00)$ & $.21(1.15)$ & & & \\
\hline Unpleasant narrative & $.00(.00)$ & $.37(1.48)$ & $\mathrm{F}<1$ & $F(1,48)=1.87, p=.18$ & $\mathrm{~F}<1$ \\
\hline
\end{tabular}

Note: TMQQ: Trauma Memory Quality Questionnaire. 
Table 4. Zero-order bivariate relationships between putative predictors of acute stress symptom severity

\begin{tabular}{|c|c|c|}
\hline Characteristic & df & CPSS \\
\hline $\operatorname{Age}^{\#}$ & 50 & .09 \\
\hline Gender $^{\mathrm{a}}$ & 50 & -.01 \\
\hline Previous mental health contact ${ }^{b}$ & 49 & .06 \\
\hline Previous trauma ${ }^{\mathrm{b}}$ & 49 & .13 \\
\hline Subjective threat of trauma ${ }^{\#}$ & 50 & $.32 *$ \\
\hline cPTCI total & 50 & $.90 * * *$ \\
\hline $\mathrm{TMQQ}^{\#}$ & 50 & .17 \\
\hline Birleson Depression" & 50 & $.74 * * *$ \\
\hline Halligan index of disorganisation ${ }^{\#}$ & 50 & $.33 *$ \\
\hline Foa positive index & 50 & -.22 \\
\hline Foa negative index & 50 & -.12 \\
\hline Foa angry index & 50 & .21 \\
\hline Foa sensory index & 50 & -.04 \\
\hline HADS Depression & 48 & $.30 *$ \\
\hline HADS Anxiety & 48 & $.32 *$ \\
\hline SDQ total problems ${ }^{\#}$ & 49 & .27 \\
\hline
\end{tabular}

Note: Associations between CPSS and other continuous variables are rho correlations. Where data are normally distributed (indicated by \#) Pearson's $r$ is quoted. The association between CPSS and gender is a point-biserial correlation. ${ }^{*} \mathrm{p}<.05,{ }^{*} * * \mathrm{p}<.001$. ${ }^{\mathrm{a}}$ Scored $1=$ male, $0=$ female; ${ }^{\mathrm{b}}$ Scored $1=$ yes, $0=$ no. 
Table 5. Linear regression model showing the prediction of continuous acute stress symptoms (CPSS scores)

Initial model adjusted $\mathrm{R}^{2}=.66, \mathrm{~F}=92.49, \quad$ Standardised $\quad \mathrm{t} \quad \mathrm{p}$

$\mathrm{df}=1,46, \mathrm{p}<.001 \quad$ coefficient $(\beta)$

\begin{tabular}{llll}
\hline cPTCI & .82 & 9.62 & $<.001 *$
\end{tabular}

Halligan index of disorganisation

$2.95 \quad .005^{*}$

HADS Anxiety

1.22

.23

HADS Depression

$.39 \quad .70$

Subjective trauma severity

$-.12$

.90

Birleson Depression

2.52

$.01^{*}$

Final model adjusted $\mathrm{R}^{2}=.71, \mathrm{~F}=58.32$,

Standardised

$\mathrm{t}$

$\mathrm{p}$

df $=2,45, \mathrm{p}<.001$ coefficient $(\beta)$

cPTCI

Halligan index of disorganisation

.79

.23

9.94

$<.001^{*}$

HADS Anxiety

2.95

$.005^{*}$

HADS Depression

1.53

.13

Subjective trauma severity

.61

.54

Birleson Depression

$-.83$

.41

1.91

.06 
Figure 1. Halligan index of disorganisation (error bars represent SEM) for the trauma and unpleasant event narratives for participants with versus without acute stress disorder (ASD)

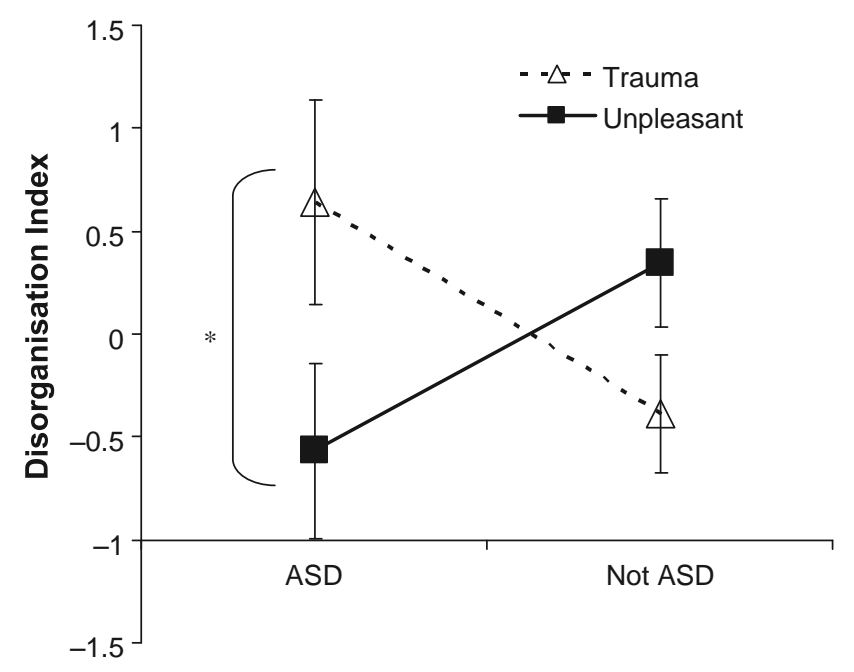

